\title{
$554-90$
}

$36953 \%$

N9 1 - 14154

Spatially Resolved Optical and Near Infrared

Spectroscopy of I $\mathrm{Zw}_{\mathrm{w}} 18$

Evan D. Skillman

Astronomy Department, University of Texas

R. C. Kennicutt

Steward Observatory, University of Arizona

$-1.25$

I MOTIVATION

For a long time, I Zw 18 has been recognized as the lowest abundance extragalactic H II region (with the possible recent exception of SBS 0335-052; Izotov 1989). As such, it is important for many studies, including the determination of the primordial helium abundance,(e.g- Davidson and Kinman 1985; and references therein). Recent imaging studies of I $\mathrm{Zw} 18$ (Davidson, Kinman, and Friedman 1989; Bufour and Hester 1989) have revealed a more complex structure to I Zw 18 than the simple two-component model previously assumed. This has given rise to concern about the reliability of chemical abundance measurements derived for I $\mathrm{Zw} 18$. We have obtained long-slit spectra covering the wavelength range $\lambda 3650$ $\lambda 10,000$, which allow us to measure physical parameters and chemical abundances as a function of position. With these new data we can investigate the SE component, which has not been studied previously, and we can address some of the concerns about abundance uncertainties.

\section{NEW OBSERVATIONS}

The spectra were obtained with 3 different telescope/instrument combinations. All observations were made using a long slit spectrograph with a 2 arcsec slit width imaged onto a TI $800 \times 800$ pixel CCD. The position angle of $-39^{\circ}$ insured that the slit covered both the NW and SE components. The three data sets are summarized in Table 1. The MMT Red Channel Echellette (Schmidt, Weymann, and Foltz 1989) makes efficient use of the full format of the CCD by cross-dispersing 5 orders (of a 20 arcsecond long slit), allowing both large wavelength coverage and intermediate resolution. The high throughput of the Red Channel combined with the MMT allowed the detection of such faint lines as [N II] $\lambda \lambda 6548,6584,[\mathrm{O}$ I] $\lambda \lambda 6300,6363$, and [S III] $\lambda \lambda 6312,9069,9532$ in both the NW and SE components (see Figure 1).

\section{III $\mathrm{N} / \mathrm{O}, \mathrm{O} / \mathrm{H}$, and $\mathrm{He} / \mathrm{H}$ in I Zw 18}

It is currently believed that while oxygen has dominantly primary nucleosynthetic origins, nitrogen benefits from both primary and secondary nucleosynthesis (cf. Pagel 1985). This is based on the observation that below $[\mathrm{O} / \mathrm{H}] \approx-0.6$, we see the primary ratio of $\log (\mathrm{N} / \mathrm{O}) \approx-1.54$, while above this $\mathrm{N} / \mathrm{O}$ increases with increasing $\mathrm{O} / \mathrm{H}$ (due to secondary nitrogen). Dufour, Garnett, and Shields (1988) presented the surprising value of $\log (\mathrm{N} / \mathrm{O})=-1.25 \pm 0.18$ for $\mathrm{I} \mathrm{Zw} 18$. This lead 
to speculation that in extreme dwarf galaxies supernovae may eject a significant fraction of their oxygen out of the galaxy, leading to a lower than expected value for the oxygen abundance. In fact, our new measurements (with a factor of 10 improvement in the $\mathrm{s} / \mathrm{n}$ ratio in the [N II] $\lambda 6584$ line) yield $\log (\mathrm{N} / \mathrm{O})=-1.5 \pm 0.1$. The N/O ratios are equal (within errors) in both components.

Campbell (1989) has proposed a radial density gradient model for I $\mathrm{Zw} 18$ in which the central density is close enough to the critical density $\left(7 \times 10^{5}\right)$ to partially collisionally de-excite the [O III] $\lambda \lambda 4959,5007$ lines. This results in a rue" oxygen abundance for $\mathrm{I} \mathrm{Zw} 18$ which is a factor of nearly 4 times higher than the generally accepted value. Figure 2 shows the density sensitive [S II] $\lambda 6717 / \lambda 6731$ ratio as a function of position across $\mathrm{I} \mathrm{Zw}$ 18. In all positions the ratio is close to the low density limit, and nowhere is there evidence of very dense gas. If the model of Campbell were correct we might expect some trace of high density gas in the [S II] ratio, such as a decrease in the ratio coincident with the continuum peak. In order for a density gradient not to effect the [S II] ratio would require that the high density region be confined to a very small fraction of the total volume of the nebula. In this case it would be visible in the imaging studies. The low value of $\mathrm{O} / \mathrm{H}$ obtained by previous studies remains secure. We derive equivalent values of $\mathrm{O} / \mathrm{H}$ (within errors) for the NW and SE components.

One of the motives of this study is to follow-up on the suggestion of Davidson and Kinman to provide additional observational material on I $\mathrm{Zw} 18$ relevant to the study of the primordial helium abundance. These new observations provide three new tests: 1.) We have high $\mathrm{s} / \mathrm{n}$ in the $\lambda 6678$ line. This is a singlet line, and should be immune from the effects of self-absorption. (previous studies concentrated on the $\lambda \lambda 5876,4471$ triplet lines); 2.) We can study the $\lambda 6678 / \lambda 6563$ line ratio as a function of position to test for the presence of neutral helium; and 3.) We have found the He II $\lambda 4686$ line (which is detected in the NW component, but not the $\mathrm{SE}$ component) to vary similarly to the Balmer emission. (The $\lambda 4686 / \lambda 4861$ ratio shows a variation of only a factor of two - centrally peaked - over the NW component, while the $\lambda 4686 /$ continuum ratio varies by more than a factor of 10 .) This implies that the He II emission is nebular, and not circumstellar. This, in turn, implies two important results. The Helium abundance should be corrected for $\mathrm{He}^{++}$(as discussed by Davidson and Kinman), and there is at least one very hot star $\left(\mathrm{T}_{\text {eff }}\right.$ $\geq 70,000 \mathrm{~K}$ ) in $\mathrm{I} \mathrm{Zw} 18$ (Bergeron, 1977).

\section{Acknowledgements}

This work was supported in part by Robert A. Welch Foundation grant F-910 to the University of Texas at Austin, and NSF grant AST-8613257 to RCK. Some of the observations reported here have been obtained at the Multiple Mirror Telescope, a joint facility of the University of Arizona and Smithsonian Institution. 


\section{References}

Bergeron, J. 1977, Ap. J., 211, 62.

Campbell, A. 1988, B.A.A.S., 20, 1038.

Davidson, K., and Kinman, T.D. 1985, Ap. J. Suppl., 58, 321.

Davidson, K., Kinman, T.D., and Friedman, S.D. 1989, A. J., in press.

Dufour, R.J., Garnett, D.R., and Shields, G.A. 1988, Ap. J., 332, 752.

Dufour, R.J., and Hester, J.J. 1989, Ap. J. (letts.), in press.

Izotov, Y. 1989, private communication.

Pagel, B.E.J. 1985, in Production and Distribution of $C, N, O$ Elements, ed. I. J.

Danziger, F. Matteucci, and K. Kjar (Munich: ESO), p.155.

Schmidt, G.D., Weymann, R.J., and Foltz, G.B. 1989, P.A.S.P, submitted.

Table 1

\begin{tabular}{llc}
\hline $\begin{array}{l}\text { Telescope/ } \\
\text { Instrument }\end{array}$ & $\begin{array}{l}\text { Wavelength } \\
\text { Coverage }\end{array}$ & Resolution \\
\hline MMT/Red Channel & $6200-6750 \AA$ & $3.5 \AA$ \\
MMT/R.C. Echellette & $4600-10,000 \AA$ & $8-15 \AA$ \\
McDonald 2.7m/LCS & $3650-5100 \AA$ & $6 \AA$ \\
\hline \hline
\end{tabular}

Figure 1.) Spectra in the region $\lambda 6500-6800$ for the SE component.

Figure 2.) The density dependent [S II] ratio as a function of position in I Zw 18 . The relative stellar continuum is plotted below for reference.
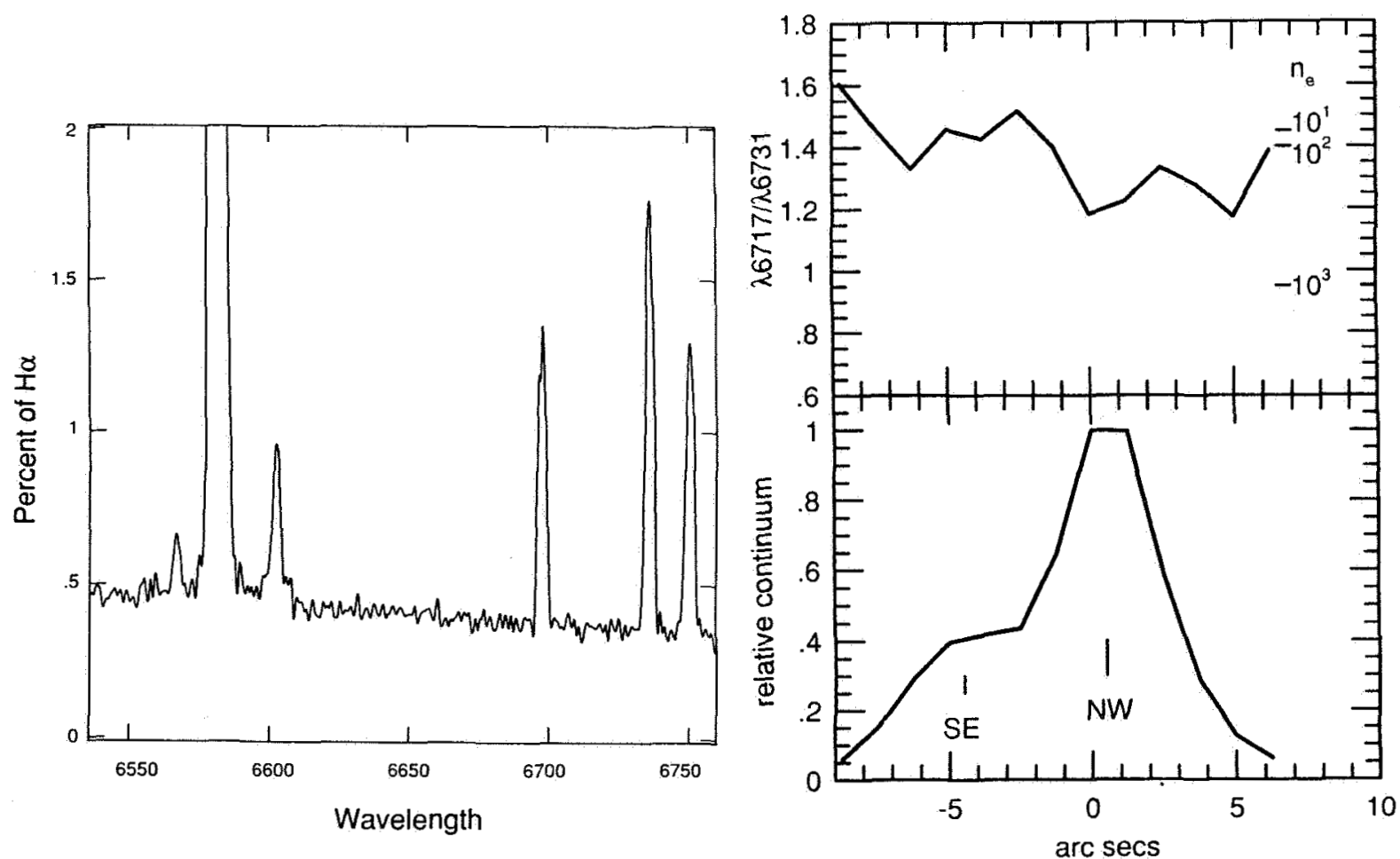